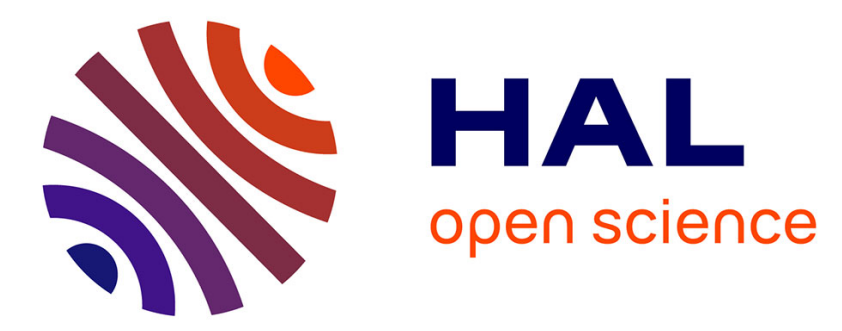

\title{
Etat structural, enracinement et alimentation hydrique du maïs. II. - Croissance et disposition spatiale du système racinaire
}

François Tardieu, Hubert Manichon

\section{- To cite this version:}

François Tardieu, Hubert Manichon. Etat structural, enracinement et alimentation hydrique du maïs. II. - Croissance et disposition spatiale du système racinaire. Agronomie, 1987, 7 (3), pp.201-211. hal-00884985

\section{HAL Id: hal-00884985 \\ https://hal.science/hal-00884985}

Submitted on 1 Jan 1987

HAL is a multi-disciplinary open access archive for the deposit and dissemination of scientific research documents, whether they are published or not. The documents may come from teaching and research institutions in France or abroad, or from public or private research centers.
L'archive ouverte pluridisciplinaire HAL, est destinée au dépôt et à la diffusion de documents scientifiques de niveau recherche, publiés ou non, émanant des établissements d'enseignement et de recherche français ou étrangers, des laboratoires publics ou privés. 


\title{
Etat structural, enracinement et alimentation hydrique du maïs. II. - Croissance et disposition spatiale du système racinaire
}

\author{
François TARDIEU \& Hubert MANICHON $(*)$
}

I.N.R.A., Laboratoire d'Agronomie INA-PG, Centre de Recherches de Paris-Grignon, F 78850 ThivervalGrignon

(*) INA-PG, Chaire d'Agronomie, F 78850 Thiverval-Grignon

Mots clés additionnels : Observation in situ des racines, tassement du sol, relations parties aériennes-parties souterraines, groupement des racines. systems.

Four trials were carried out in the field to study the effect on the maize root system of the structure of the ploughed layer. In each case the experimental treatments were the 3 typical ploughed layer structures $\mathrm{O}, \mathrm{B}$ and $\mathrm{C}$ presented in the first article of this series. Treatments affected evolution with time of the depth of the root front, but not maximum root depth measured after silking. Total root weight increased differently between treatments, but root/top + root weight ratios were similar in all situations. Growth of the whole plant (roots and tops) was accordingly faster in treatment $O$ than in $B$ and $C$. Root densities measured at flowering were significantly different in the ploughed layer, where soil structure differs between treatments, but also in the non-tilled layers where it does not. Over the whole profile, the horizontal and vertical spatial distribution of roots was markedly affected by the ploughed layer structure. This can be explained by the spatial variability, at the decimetre scale of mechanical resistance to penetration in the ploughed layer ; in non-tilled layers of treatment $C$, the irregularity of root patterns was due to localized reductions of root density under obstacles located in the ploughed layer ("shadow effect").

Additional key words : In situ observation of roots, soil compaction, top-root growth relations, root clumping.

\section{LISTE DES ABRÉVIATIONS}

Horizon de la couche labourée, affiné lors du travail superficiel.
Horizon non affiné de la couche labourée. Traitement expérimental : H5 de structure fragmentaire, formée de terre fine.
Traitement expérimental : H5 de structure fragmentaire formée de mottes de plusieurs $\mathrm{dm}^{3}$, et de cavités structurales.

Traitement expérimental : H5 de structure continue et compacte.

Etat interne des mottes : porosité structurale très réduite. 
$\Gamma \quad$ Etat interne des mottes, porosité structurale importante.

G82, G83, G85, M82 : Essais effectués à Grignon (G) ou à Montmirail (M) en 1982, 1983 ou 1985.

$\mathrm{n}$ « $\mathrm{f}$ » Stade phénologique « $\mathrm{n}$ feuilles visibles ».

$r, F, C V$ Coefficient de corrélation, F calculé de FISHER et coefficient de variation (écart type/moyenne).

$>\quad$ Différence significative, comparaison de moyennes par le « $\mathrm{T} »$ de BONFERRONI, risque global 5 p. 100.

\section{INTRODUCTION}

Les relations entre l'état structural du sol et l'enracinement ont souvent été présentées dans la littérature à travers la modélisation de la pénétration des racines dans des volumes de petites dimensions, formés soit de matériaux artificiels (WIERSUM, 1957; AUBERTIN \& KARDOS, 1965 ; SCOTT-RUSSEL \& GOSS, 1974), soit de terre remaniée (TAYLOR \& GARDNER, 1963 ; BARLEY, 1963 ; MAERTENS, 1964b, notamment). Dans ces conditions, ces travaux montrent les effets respectifs, d'une part de la porosité du milieu et des caractéristiques géométriques des pores, d'autre part de la rigidité de ce milieu, liée s'il s'agit de terre, à son potentiel hydrique. Ceci ouvre la voie à l'élaboration de modèles déterministes de la trajectoire des racines (DEXTER \& HEWITT, 1978 ; HewitT \& DEXTER, 1984).

L'utilisation de ces résultats pour rendre compte de l'effet de l'état physique du sol sur les systèmes racinaires au champ (SEBILlOTTE, 1963 ; BARBER, 1971 ; RAGHAVAN et al., 1979) se heurte à des difficultés liées à la nature différente des objets étudiés au champ et au laboratoire :

- Dans les expériences de laboratoire que nous avons citées, le volume de sol dans lequel se développent les racines est entièrement affecté par le tassement, ce qui n'est pas le cas au champ, où les horizons tassés ne représentent qu'une faible partie du volume potentiellement enraciné par le maïs (TARDIEU \& MANICHON, 1987). D'autre part, au champ ce volume de sol subit au cours du temps une variation d'état physique, en particulier de teneur en eau et de degré de fissuration, alors que tel n'est pas le cas dans les expériences de laboratoire réalisées en temps court.

- Dans ces expériences de laboratoire, les plantes dont le système racinaire est étudié sont placées dans des conditions thermiques et alimentaires optimales, tandis que les systèmes racinaires au champ appartiennent à des peuplements végétaux qui peuvent subir des stress de croissance. Or ces stress se répercutent sur la vitesse de croissance du système racinaire (BROUWER, 1966 ; WeLBANK et al., 1974), et probablement sur le rythme d'apparition des racines (PICARD et al., 1985). Si certains états structuraux de la couche labourée ont pour conséquence des conditions thermiques défavorables ou une alimentation hydrique et minérale insuffisante par rapport aux besoins du peuplement végétal, des relations autres que celles liées à la seule résistance mécanique à la pénétration peuvent exister entre l'état structural et l'enracinement.

A l'étude de l'enracinement des peuplements végétaux des traitements $\mathrm{O}, \mathrm{B}$ et $\mathrm{C}$, définis dans le $\mathbf{1}^{\text {er }}$ arti- cle de cette série (TARDIEU \& MANICHON, 1987), nous avons donc adjoint d'une part une étude de l'alimentation hydrique du maïs, d'autre part le suivi de la croissance des parties aériennes des peuplements.

\section{MATÉRIEL ET MÉTHODES}

Les essais ont été réalisés dans la région parisienne, à Grignon (Yvelines) en 1982, 83 et 85 , et à Montmirail en 1982. Les caractéristiques des parcelles et le dispositif expérimental sont décrits dans le $1^{\text {er }}$ article de cette série (TARDIEU \& MANICHON, 1987). Le maïs (hybride simple LG1) a été semé début mai à une densité de 87000 plantes/ha; 100 unités de $\mathrm{P}_{2} \mathrm{O}_{5}$ et $\mathrm{K}_{2} \mathrm{O}$ ont été apportées en automne, et 140 unités d'azote au semis. Le désherbage et le contrôle phytosanitaire ont été effectués selon les normes locales de culture.

L'enracinement a été suivi entre la levée et une semaine après la floraison, période pendant laquelle le système racinaire du maïs est en croissance (FoTH, 1962 ; MENGEL \& BARBER, 1974 ; VARLETGRANCHER, 1982). Les dates d'observations ont été choisies en fonction du stade phénologique des peuplements végétaux. Pour ce faire, on a compté le nombre de feuilles visibles tous les $3 \mathrm{j}$ sur 30 plantes par traitement, sauf à M82 où la fréquence était de $10 \mathrm{j}$. Un léger retard de développement étant apparu chaque année en $\mathrm{B}$ et $\mathrm{C}$ par rapport à $\mathrm{O}$ (tabl. 1), les comparaisons entre traitements ont été faites à même stade, et non à même date, afin de ne pas confondre d'éventuelles différences de croissance du système racinaire avec les écarts de vitesse de développement. Six stades ont été étudiés à G83 (4 en B) ; à G82, M82 et G85, les observations n'ont été effectuées qu'à la floraison. Dans cette dernière situation, elles n'ont concerné que les traitements $\mathrm{O}$ et $\mathrm{C}$ où nous voulions confirmer les résultats obtenus lors des années antérieures. Les résultats présentés ici concernent surtout la campagne G83, ceux obtenus à la floraison des 3 autres essais permettant de renforcer les conclusions obtenues.

Dans les placettes étudiées, de $30 \mathrm{~cm}$ parallèlement au rang de semis sur $80 \mathrm{~cm}(40 \mathrm{~cm}$ de part et d'autre du rang), les parties aériennes ont d'abord été coupées, séchées $48 \mathrm{~h}$ à $80^{\circ} \mathrm{C}$ et pesées (une courbe de croissance a par ailleurs été établie sur un plus grand nombre de placettes (TARDIEU, 1984). Le système racinaire a été caractérisé par la méthode décrite dans un article précédent (TARDIEU \& MANICHON, 1986 $b$ ) :

- Les impacts de racines ont été cartographiés avec un maillage de $2 \times 2 \mathrm{~cm}$ sur un plan vertical et, dans un sous-échantillon de placettes, sur 5 plans horizontaux superposés. Ces cartes ont été faites sur les mêmes plans que les cartes structurales, ce qui permet une mise en correspondance à l'échelle de la case entre l'état structural et la densité racinaire.

- La caractérisation des systèmes racinaires en tant que capteur d'eau a été faite par 2 méthodes. Sur les placettes où des cartographies horizontales ont été effectuées, on a calculé sur chaque plan la fonction de répartition des distances entre chaque point du sol et la racine la plus proche (TARDIEU \& MANICHON, 1986b). Lorsque la cartographie racinaire a été faite sur un plan vertical, cette caractérisation a été obtenue par un indice synthétique ; celui-ci est égal au nombre de cases 
TABLEAU 1

Dates et effectifs des observations de l'enracinement par la méthode de cartographie racinaire sur plan vertical.

(*) Réalisation de 5 cartographies horizontales sur les mêmes placettes.

Dates and number of observations of root system with the vertical root mapping method.

(*) 5 horizontal mappings carried out on the same sample.

\begin{tabular}{|c|c|c|c|c|c|c|}
\hline & \multicolumn{2}{|c|}{$\mathrm{O}$} & \multicolumn{2}{|c|}{ B } & \multicolumn{2}{|c|}{$\mathrm{C}$} \\
\hline & Date & Effectif & Date & Effectif & Date & Effectif \\
\hline \multicolumn{7}{|l|}{$G 82$} \\
\hline Floraison +1 semaine & $20 / 7$ & 12 & $22 / 7$ & 8 & $23 / 7$ & 8 \\
\hline \multicolumn{7}{|l|}{ M82 } \\
\hline Floraison +2 semaines & $2 / 8$ & 4 & $3 / 8$ & 4 & $5 / 8$ & 8 \\
\hline \multicolumn{7}{|l|}{$G 83$} \\
\hline \multicolumn{7}{|l|}{ Stades } \\
\hline 6 feuilles & $10 / 6$ & 4 & $10 / 6$ & 4 & $13 / 6$ & 4 \\
\hline 8 feuilles & $17 / 6$ & 3 & $21 / 6$ & 4 & $22 / 6$ & 4 \\
\hline $9-10$ feuilles & $21 / 6$ & 4 & - & - & $28 / 6$ & 3 \\
\hline 11 feuilles & $29 / 6$ & 7 & $1 / 7$ & 4 & $4 / 7$ & 4 \\
\hline Floraison +1 semaine & $22 / 7$ & 7 & $23 / 7$ & 12 & $27 / 7$ & 10 \\
\hline Floraison +3 semaines $\left({ }^{*}\right)$ & $3 / 8$ & 4 & - & - & $8 / 8$ & 3 \\
\hline \multicolumn{7}{|l|}{$G 85$} \\
\hline Floraison +1 semaine & $23 / 7$ & 4 & - & - & $28 / 7$ & 2 \\
\hline
\end{tabular}

de la carte dont le centre de gravité se trouve à moins de $4 \mathrm{~cm}$ de l'impact de racine le plus proche, si cette racine est en contact avec la terre environnante. Cet indice est une estimation, sur le plan considéré, de la surface du plan dont les points sont à moins de $4 \mathrm{~cm}$ d'un impact. Il permet de classer entre placettes le volume de sol situé à proximité d'une racine en contact avec la terre.

- Une évaluation de la masse racinaire peut être obtenue à partir des cartes verticales, en sommant les notes de densité racinaire linéarisées par rapport à la masse racinaire (TARDIEU \& MANICHON, 1986b).

A G82 et 83 , la profondeur d'enracinement a été suivie en continu par l'observation endoscopique des racines au travers de tubes transparents implantés dans le sol, suivant la procédure mise au point par MAERTENS \& Clauzel (1982). De 4 à 6 tubes ont été implantés par traitement, avec une inclinaison de $30^{\circ}$ par rapport à la verticale.

Les résultats ont été traités par analyse de variance à niveau de risque 5 p. 100 ; il n'y a pas d' « effet bloc », en accord avec l'homogénéité pédologique des parcelles d'essai. Les comparaisons multiples entre traitements ont été effectuées par la méthode du T de BONFERRONI, à niveau global de risque 5 p. 100 . Lors des mises en correspondance de 2 variables, les nuages de points ont été traités par régression, puis par analyse de covariance, ceci afin de tester si les pentes et les ordonnées à l'origine des 3 nuages $\mathrm{O}, \mathrm{B}$ et $\mathrm{C}$ sont différentes.

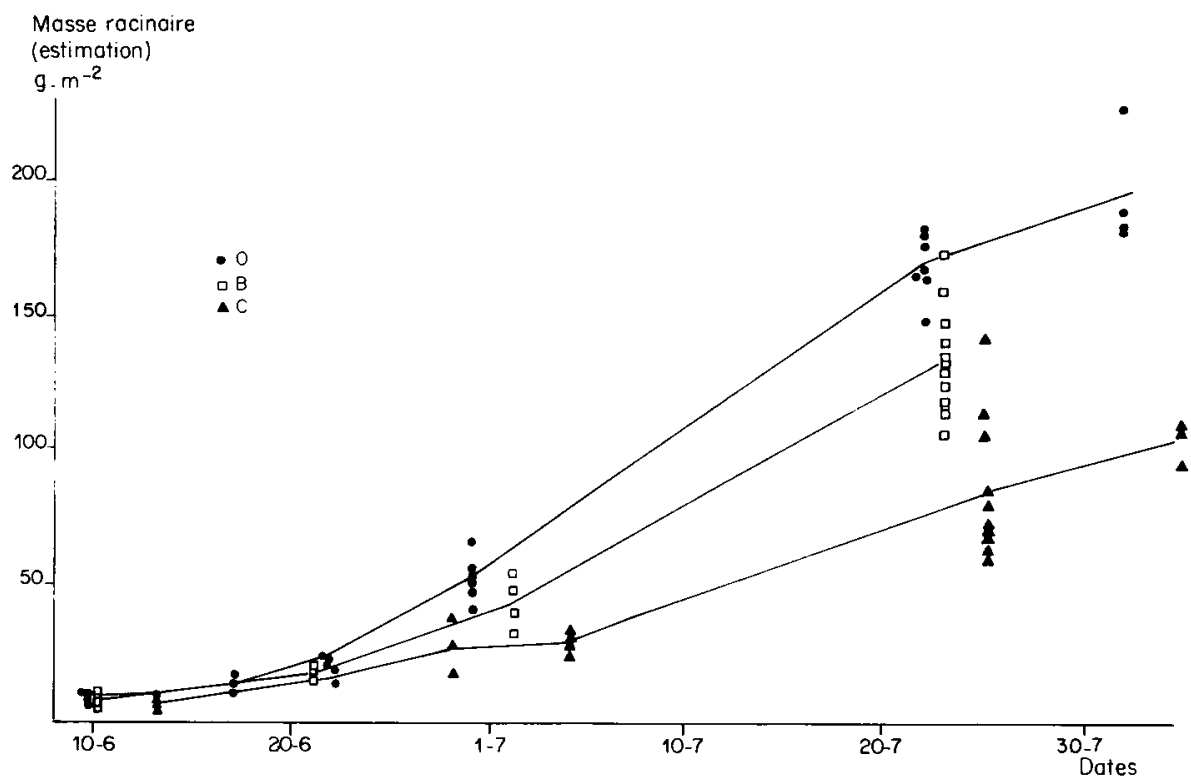

Figure 1

Croissance du système racinaire, Grignon 1983. Chaque point représente une placette observée, les traits continus relient les moyennes correspondant à chaque date.
Root system growth, Grignon 1983. Each point represents one root map; plain lines link mean values at each date. 


\section{RÉSULTATS}

\section{A. Mise en place du système racinaire}

\section{Croissance de la masse racinaire}

L'évolution dans le temps des masses racinaires estimées est présentée à la figure 1 pour l'essai G83. Bien que leur méthode de calcul ne soit pas directe, les courbes obtenues en $\mathrm{O}$ et $\mathrm{B}$ sont semblables à d'autres obtenues par pesée : leur forme et les niveaux atteints sont comparables à ceux présentés par FOTH (1962), MAERTENS (1964a), VARLET-GRANCHER (1982) et LUBET \& JUSTE (1985). La croissance, assez lente au début du cycle, s'accélère à partir de la montaison (Stade $8 \mathrm{f}$ ), moment où l'augmentation de masse des parties aériennes devient également rapide. Ce stade est atteint les 17,21 et 22/06, respectivement en O, B et C. Une différence significative entre les traitements apparaît alors, le classement étant $\mathrm{O}>\mathrm{B}>\mathrm{C}$. Ce classement persiste jusqu'à la floraison (tabl. 2), et est le même dans les autres situations expérimentales (des différences significatives ne sont mises en évidence qu'à G82 et 83 , en raison des nombres insuffisants de répétitions dans les 2 autres situations). Le tableau 2 fait aussi apparaître que les coefficients de variation (CV) sont plus élevés pour $\mathrm{C}$ que pour les 2 autres traitements : on y a observé, à quelques mètres de distance, des placettes de masses racinaires très contrastées. Les CV sont, par ailleurs, plus bas que ceux couramment observés avec d'autres méthodes d'ëtude de l'enracinement (cf. Discussion).

2. Rapport masse racinaire-matière sèche totale à la floraison

\section{a) Comparaison entre traitements}

Les rapports masse racinaire sur matière sèche totale (masse racinaire + masse aérienne des mêmes placet- tes) sont proches entre traitements, et entre situations expérimentales (tabl. 2), bien que les masses racinaires varient du simple au double. Ils sont compris entre 15 et $21 \mathrm{p} .100$, ce qui est de l'ordre de ceux calculés à partir des données de VARLET-GRANCHER (1982) et de LUBET \& JUSTE (1985).

\section{b) Variabilités inter et intra-traitement}

La figure 2 présente la mise en correspondance des masses racinaires évaluées à la floraison de l'essai G83 avec les masses aériennes mesurées à la même date et sur les mêmes placettes. Une corrélation étroite

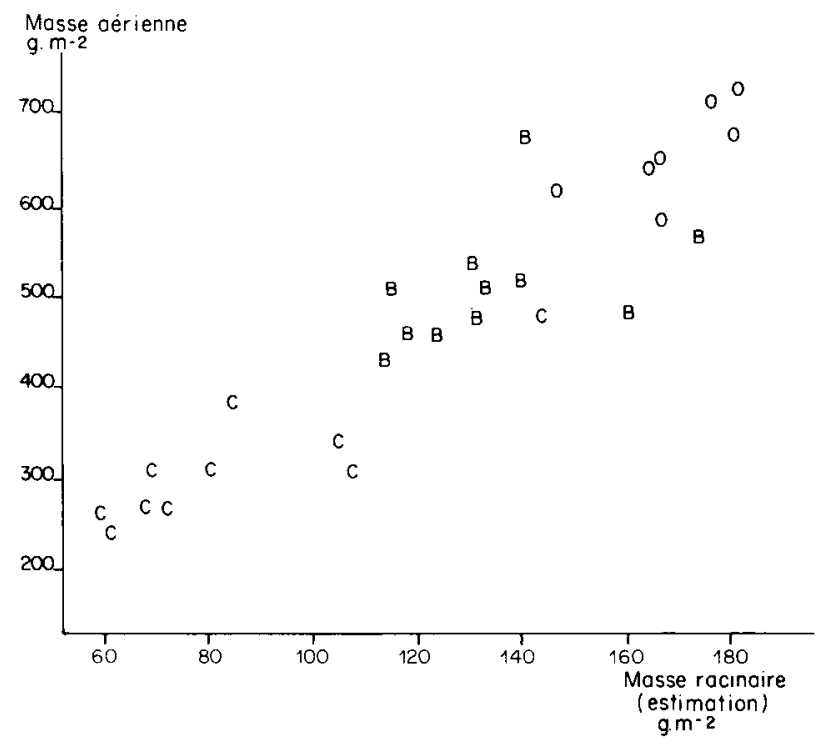

Figure 2

Mise en correspondance des masses souterraines (estimation) et aériennes des placettes étudiées. Floraison, Grignon 1983. Chaque placette est figurée par le nom du traitement auquel elle appartient. Root weight (estimated) plotted against shoot weight, for each area sample $(30 \times 80 \mathrm{~cm})$ at silking, Grignon 1983. (Each area sample is marked with the name of the treatment to which it belongs.)

TABLEAU 2

Matière sèche de racines (estimation), matière sèche racinaire/matière sèche totale (MSR/MST) et profondeur d'enracinement à la floraison. $x$ : moyenne ; $C V$ : coefficient de variation. A l'intérieur d'une ligne, les moyennes suivies par une même lettre ne sont pas significativement différentes au seuil $5 \%$ d'après le test de comparaison multiple de Bonferroni.

Root dry matter (estimation), root/total dry matter mass (MSR/MST) and rooting depth at silking. $x:$ average; CV : coefficient of variation. Means in horizontal lines followed by the same letter are not significantly different according to the Bonferroni multiple comparison test.

\begin{tabular}{|c|c|c|c|c|c|c|c|c|c|}
\hline & \multicolumn{3}{|c|}{$\begin{array}{l}\text { Estimation de la masse totale de } \\
\text { racines } \mathrm{g} \cdot \mathrm{m}^{-2}\end{array}$} & \multicolumn{3}{|c|}{ Rapport MSR/MST } & \multicolumn{3}{|c|}{$\begin{array}{l}\text { Profondeur maximale d'enracinement } \\
\mathrm{cm}\end{array}$} \\
\hline & $\mathrm{O}$ & B & $\mathrm{C}$ & $\mathrm{O}$ & B & $\mathrm{C}$ & $\mathrm{O}$ & B & $\mathrm{C}$ \\
\hline $\begin{array}{l}\mathrm{G} 82 \\
\mathrm{x} \\
\mathrm{CV}(\%)\end{array}$ & $\begin{array}{c}122 \\
18\end{array}$ & $\begin{array}{l}110 \mathrm{ab} \\
19\end{array}$ & $\begin{array}{l}81 \mathrm{~b} \\
22\end{array}$ & $\begin{array}{l}15,7 \mathrm{a} \\
15\end{array}$ & $\begin{array}{l}18,5 \mathrm{a} \\
13\end{array}$ & $\begin{array}{l}15,9 \mathrm{a} \\
12\end{array}$ & $\begin{array}{c}82 \mathrm{a} \\
5\end{array}$ & $\begin{array}{c}76 \mathrm{~b} \\
5\end{array}$ & $\begin{array}{c}73 \mathrm{~b} \\
6\end{array}$ \\
\hline $\begin{array}{l}\text { M82 } \\
x \\
\mathrm{CV}(\%)\end{array}$ & $\begin{array}{c}145 \mathrm{a} \\
10\end{array}$ & $\begin{array}{c}113 \mathrm{a} \\
17\end{array}$ & $\begin{array}{c}103 \mathrm{a} \\
28\end{array}$ & $\begin{array}{c}16,7 \mathrm{a} \\
6\end{array}$ & $\begin{array}{l}14,7 \mathrm{a} \\
17\end{array}$ & $\begin{array}{l}15,7 \mathrm{a} \\
15\end{array}$ & $\begin{array}{c}93 \mathrm{a} \\
5\end{array}$ & $\begin{array}{c}80 \mathrm{a} \\
8\end{array}$ & 85 a \\
\hline $\begin{array}{l}\text { G83 } \\
x \\
C V(\%)\end{array}$ & $\begin{array}{c}169 \text { a } \\
7\end{array}$ & $\begin{array}{c}132 b \\
15\end{array}$ & $\begin{array}{l}85 \mathrm{c} \\
31\end{array}$ & $\begin{array}{c}20,2 \mathrm{a} \\
5\end{array}$ & $\begin{array}{c}20,9 \text { a } \\
9\end{array}$ & $\begin{array}{c}20,9 \mathrm{a} \\
8\end{array}$ & $\begin{array}{c}101 \text { a } \\
7\end{array}$ & $\begin{array}{c}92 \mathrm{~b} \\
8\end{array}$ & $\begin{array}{c}90 \mathrm{~b} \\
4\end{array}$ \\
\hline $\begin{array}{l}\text { G85 } \\
x \\
C V(\%)\end{array}$ & $\begin{array}{r}153 \\
6\end{array}$ & & 92 & $\begin{array}{c}15,7 \\
5\end{array}$ & & $\begin{array}{r}14,8 \\
-\end{array}$ & $\begin{array}{r}105 \\
5\end{array}$ & & $\stackrel{92}{-}$ \\
\hline
\end{tabular}


$(r=0,93)$ existe entre ces 2 variables. Une analyse de covariance a été effectuée sur ces données, la masse racinaire étant mise en relation avec le traitement expérimental et la covariable matière sèche aérienne. Cette analyse montre l'absence d'interaction traitementcovariable ; on peut donc considérer qu'il existe une pente commune aux 3 nuages $\mathrm{O}, \mathrm{B}$ et $\mathrm{C}$. La covariable ainsi définie rend compte de l'effet traitement : celui-ci n'apparaît plus alors comme significatif ( $F$ calculés de 0,2 et 21,0 pour le traitement et la covariable respectivement ; CV résiduel de l'analyse : 12 p. 100).

La variabilité de la masse racinaire, intra et intertraitements, est donc étroitement liée à celle de la masse aérienne. Il existe à l'intérieur des champs d'essai une variabilité de la matière sèche totale, qui
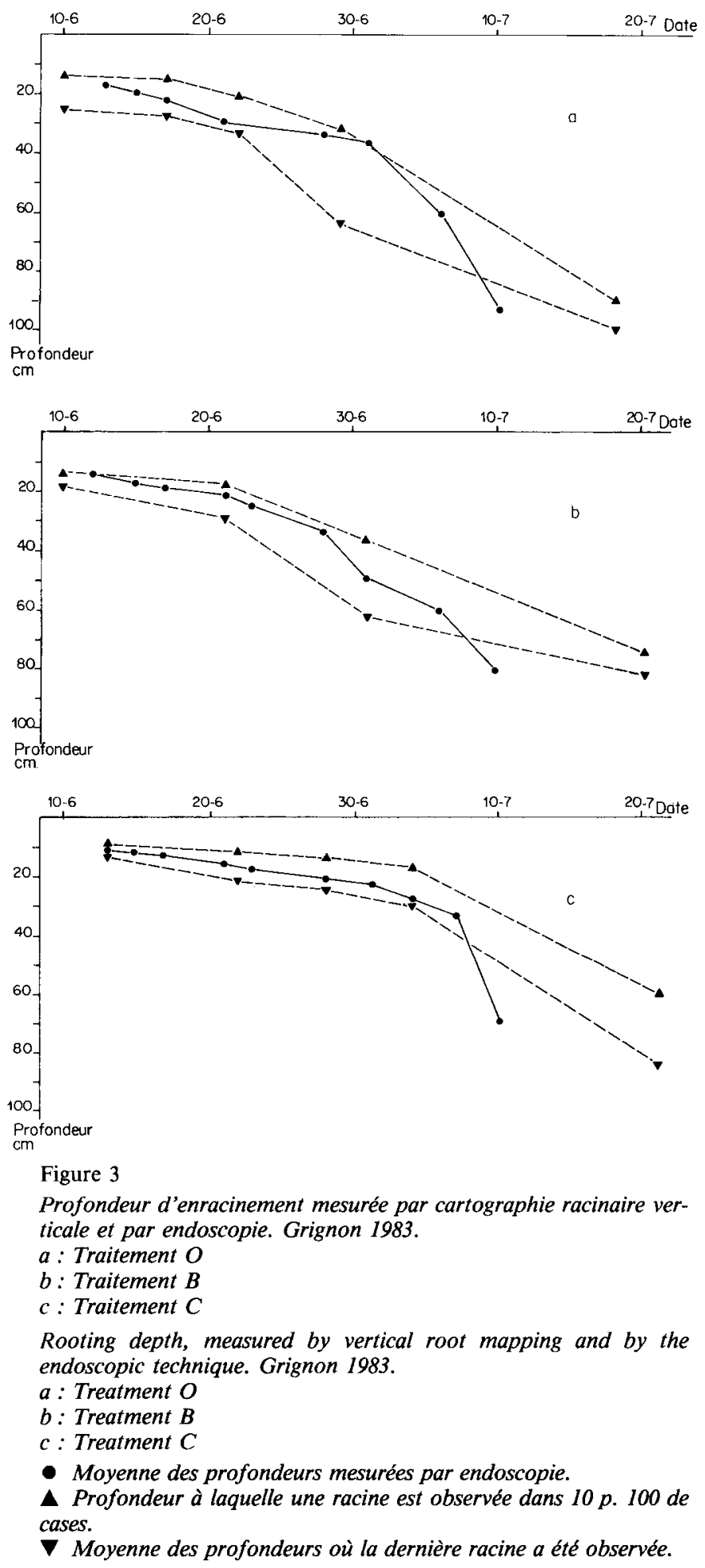

s'exprime à la fois sur les parties aériennes et souterraines des peuplements végétaux.

\section{Profondeur d'enracinement (fig. 3)}

a) Dans les traitements $\mathrm{O}$ et $\mathrm{B}$, des racines ont franchi le fond de la couche labourée aux environs du stade $9 \mathrm{f}$ en 1983, stade atteint les 21 et $25 / 06$ en $\mathrm{O}$ et B respectivement. Les observations endoscopiques de G82 fournissent des indications convergentes. Certaines racines, peu nombreuses, se sont alors allongées très rapidement (approfondissement du front racinaire de $30 \mathrm{~cm}$ environ en 8 jours, ce qui correspond aux vitesses maximales observées par JORDAN, 1986) à l'intérieur de galeries verticales de vers de terre. Cet aspect de la dynamique racinaire, observé sur profil, n'a pas été révélé par les observations endoscopiques.

Début juillet, un défaut d'ajustement s'est produit entre le sol et les tubes d'accès de l'endoscope, consécutif à un retrait de la terre après dessiccation. Les racines se sont alors comportées; dans la cavité ainsi ménagée, comme dans les galeries de vers de terre, et ont atteint rapidement la profondeur maximale d'enracinement.

b) Dans le traitement $\mathrm{C}$, la presque totalité des racines est restée confinée, jusqu'au début de juillet, dans l'horizon ameubli par le travail superficiel (horizon H1). Certaines ont pénétré à partir de cette date dans des fentes de retrait apparues à ce moment (TARDIEU \& MANICHON, 1987). Il est à noter que la discontinuité liée à l'implantation du tube de l'endoscope a eu le même rôle que ces dernières ; les profondeurs mesurées par endoscopie tendent ici à être égales aux profondeurs maximales d'enracinement, ce qui n'est pas le cas dans les traitements $O$ et $B$. A partir du début juillet, les racines ont atteint les couches non tassées, et l'approfondissement du front racinaire a été rapide.

c) A la floraison les différences de profondeur d'enracinement entre traitements ne sont plus que de l'ordre de $10 \mathrm{~cm}$ dans les 4 essais réalisés (tabl. 2). Les différences entre les traitements $O$ d'une part, $B$ et $C$ d'autre part sont cependant significatives à G82 et G83. A G85, la réalisation de plans horizontaux à $1,20 \mathrm{~m}$ de profondeur a montré que les racines n'atteignaient celle-ci dans aucun des traitements.

\section{B. Disposition spatiale du système racinaire et con- tacts terre-racine à la floraison}

\section{Distribution verticale de la densité racinaire}

a) La figure 4 présente pour G83 la relation entre la profondeur et la densité racinaire, caractérisée par la proportion de cases où un impact de racine au moins a été observé. Les courbes correspondant aux traitements $\mathrm{O}$ et $\mathrm{B}$ ont des formes similaires, avec des valeurs plus élevées en $\mathrm{O}$ qu'en $\mathrm{B}$. La densité augmente avec la profondeur dans les 10 premiers $\mathrm{cm}$, elle décroît ensuite quasi-linéairement en $O$, avec une légère dépression entre la couche labourée et l'horizon sousjacent ; en $\mathrm{B}$, cette décroissance ne commence qu'à $40 \mathrm{~cm}$. Mis à part l'augmentation de la densité avec la profondeur dans les 10 premiers $\mathrm{cm}$ (liée à la noncolonisation de l'inter-rang tassé par la roue du tracteur qui a effectué le semis), la forme de ces courbes 

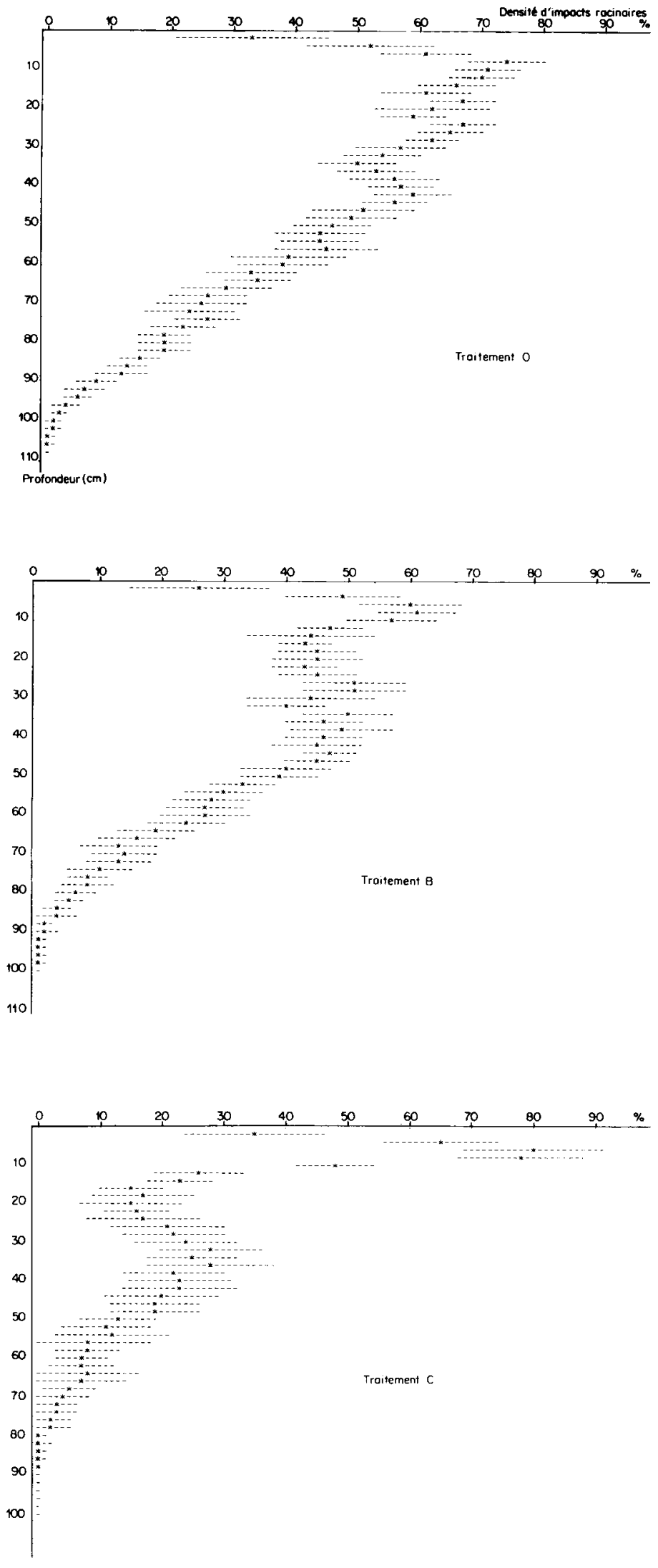

Figure 4

Moyenne (*) - mean (*).

Moyenne et intervalle de confiance (.05) par classe de profondeurs de la proportion de cases de $2 \times 2 \mathrm{~cm}$ où un impact de racine au moins a été observé. Floraison, Grignon 1983.

Mean and confidence interval (.05) of the proportion of $2 \times 2 \mathrm{~cm}$ squares where at least one root impact was observed. Silking, Grignon 1983. est similaire à celles trouvées dans la littérature (FOTH, 1962 ; MENGEL \& BARBER, 1974 ; AlLMARAS et al., 1975). En revanche, elles ont une forme différente dans le traitement $\mathrm{C}$ : on observe une densité comparable à celle de $\mathrm{O}$ dans l'horizon $\mathrm{H} 1$, une diminution brutale de la densité à l'interface entre cet horizon et l'horizon H5. La colonisation des couches sousjacentes, bien que légèrement plus intense que celle de $\mathrm{H} 5$, est très inférieure à celle constatée en $\mathrm{O}$ et $\mathrm{B}$.

\section{b) Densités moyennes par horizon (tabl. 3)}

Dans $\mathrm{H} 1$ celles-ci sont proches entre traitements ; elles sont, au contraire, significativement différentes dans H5, où l'état structural est affecté par les traitements, mais aussi dans les couches non travaillées où il ne l'est pas (TARDIEU \& MANICHON, 1987). Une corrélation étroite, semblable à celle présentée à la figure 2 , existe entre les valeurs observées dans les couches non travaillées en 1983 et la matière sèche aérienne des mêmes placettes $(r=0,93)$. L'analyse de covariance montre, là aussi, que la pente est la même pour les 3 nuages de points $\mathrm{O}, \mathrm{B}$ et $\mathrm{C}$; la covariable matière sèche aérienne rend compte de la quasi-totalité des différences entre traitements, malgré un effet spécifique de ceux-ci à la limite de la signification (F calculés : 3,3 et 19,0 respectivement pour le traitement et la covariable ; $\mathrm{CV}$ résiduel de 16 p. 100). En revanche dans H5, l'effet spécifique des traitements est significatif : à matières sèches aériennes égales, le classement des densités dans cet horizon est $\mathrm{O}>\mathrm{B}>\mathrm{C}$ ( $\mathrm{F}$ calculés de 8,7 et 129,$0 ; C V$ résiduel de 13 p. 100).

\section{Disposition horizontale des racines et contact terre- racine}

L'étude détaillée de la disposition horizontale des racines est présentée, pour G83, dans un précédent article (TARDIEU \& MANICHON, 1986 b) ; nous nous bornerons ici à en généraliser les résultats aux autres situations expérimentales.

\section{a) Horizon $\mathrm{H5}$}

Le tableau 4 montre que, dans cet horizon, les racines ont colonisé préférentiellement les zones de faible résistance mécanique à la pénétration (zones fragmentaires et fissurées). Les zones massives d'état interne $\Delta$ sont très peu pénétrées par les racines, quelle que soit l'année. Ceci concerne le traitement B de G82, M82 et G83, ainsi que le traitement $\mathrm{C}$ de M82, G83 et 85 . Dans le traitement $\mathrm{C}$ de G82, où les zones massives sont de type $\Gamma$, celles-ci sont relativement mieux colonisées. La disposition spatiale des racines (TARDIEU \& MANICHON, 1986a) est donc plus groupée en B et en $C$, où les zones enracinées représentent une faible proportion de l'horizon $\mathrm{H} 5$, qu'en $\mathrm{O}$ où la majeure partie de cet horizon est colonisée.

D'autre part, la proportion de cases où les racines sont en contact direct avec la terre environnante a été évaluée dans chacun des traitements. Dans le traitement $\mathrm{O}$, ce type de contact concerne la quasi-totalité des racines (tabl. 4). Dans le traitement C de M82, G83 et $\mathrm{G} 85$, où l'état interne des mottes est de type $\Delta$, il est peu fréquent : la plus grande partie des racines est à l'intérieur de fentes de retrait et, de ce fait, non adhérentes à la terre. Dans le traitement $\mathrm{B}$, une proportion 
TABLEAU 3

Densités moyennes par horizon : proportion de cases où un impact de racine au moins a été observé (floraison). Proportion (\%) of squares per layer where at least one root impact was observed (silking).

\begin{tabular}{|c|c|c|c|c|c|c|c|c|c|}
\hline & \multicolumn{3}{|c|}{$\begin{array}{c}\text { Horizon } \\
\text { H1 }\end{array}$} & \multicolumn{3}{|c|}{$\begin{array}{c}\text { Horizon } \\
\text { H5 }\end{array}$} & \multicolumn{3}{|c|}{$\begin{array}{c}\text { Couches } \\
\text { non travaillées }\end{array}$} \\
\hline & O & B & C & o & B & C & o & B & $\mathrm{C}$ \\
\hline $\begin{array}{l}\mathrm{G} 82 \\
\mathrm{x} \\
\mathrm{CV}(\%)\end{array}$ & $\begin{array}{l}49,5 \mathrm{a} \\
23\end{array}$ & $\begin{array}{l}35,0 \mathrm{a} \\
17\end{array}$ & $\begin{array}{l}41,5 \text { a } \\
36\end{array}$ & $\begin{array}{l}69,2 \mathrm{a} \\
14\end{array}$ & $\begin{array}{l}57,2 \mathrm{ab} \\
17\end{array}$ & $\begin{array}{l}45,3 \mathrm{~b} \\
18\end{array}$ & $\begin{array}{l}25,0 \mathrm{a} \\
12\end{array}$ & $\begin{array}{l}20,8 \text { a } \\
25\end{array}$ & $\begin{array}{l}14,4 \mathrm{~b} \\
28\end{array}$ \\
\hline $\begin{array}{l}\text { M82 } \\
x \\
\text { CV }(\%)\end{array}$ & $\begin{array}{c}74,0 \mathrm{a} \\
5\end{array}$ & $\begin{array}{l}57,0 \mathrm{a} \\
10\end{array}$ & $\begin{array}{l}65,0 \mathrm{a} \\
10\end{array}$ & $\begin{array}{l}59,7 \mathrm{a} \\
15\end{array}$ & $\begin{array}{l}24,4 \mathrm{a} \\
14\end{array}$ & $\begin{array}{l}15,7 \mathrm{a} \\
33\end{array}$ & $\begin{array}{l}24,4 \text { a } \\
13\end{array}$ & $\begin{array}{l}21,2 \mathrm{ab} \\
18\end{array}$ & $\begin{array}{l}15,7 \mathrm{~b} \\
37\end{array}$ \\
\hline $\begin{array}{l}\text { G83 } \\
x \\
\text { CV }(\%)\end{array}$ & $\begin{array}{l}59,5 \mathrm{a} \\
17\end{array}$ & $\begin{array}{l}52,0 \mathrm{a} \\
18\end{array}$ & $\begin{array}{l}62,5 \text { a } \\
18\end{array}$ & $\begin{array}{c}65,8 \mathrm{a} \\
8\end{array}$ & $\begin{array}{c}47,5 \mathrm{~b} \\
9\end{array}$ & $\begin{array}{l}20,8 \mathrm{c} \\
36\end{array}$ & $\begin{array}{l}33,4 \mathrm{a} \\
14\end{array}$ & $\begin{array}{l}23,2 \mathrm{~b} \\
21\end{array}$ & $\begin{array}{l}10,5 \mathrm{c} \\
58\end{array}$ \\
\hline $\begin{array}{l}\text { G85 } \\
x \\
\text { CV }(\%)\end{array}$ & $\begin{array}{c}64,0 \\
6\end{array}$ & & $\begin{array}{r}67,0 \\
-\end{array}$ & $\begin{array}{c}66,7 \\
5\end{array}$ & & $\stackrel{13,6}{-}$ & $\begin{array}{l}29,7 \\
11\end{array}$ & & $\frac{12,4}{-}$ \\
\hline
\end{tabular}

\section{TABLEAU 4}

Colonisation de l'horizon H5 et catégories structurales. Proportion de cases où un impact de racine au moins a été observé. * : effectif de cases nul, ou trop faible pour qu'une densité soit calculée.

Colonization of the H5 layer and soil structure. Proportion of squares where at least one root impact was observed. * : null or insufficient number of squares to allow the calculation of density.

\begin{tabular}{|c|c|c|c|c|c|c|}
\hline & \multicolumn{5}{|c|}{ Types d'état structural } & \multirow{2}{*}{$\begin{array}{c}\% \\
\text { cases ou les } \\
\text { racines sont } \\
\text { en contact avec } \\
\text { la terre }\end{array}$} \\
\hline & $\begin{array}{c}\text { Fragm. } \\
\text { terre } \\
\text { fine }\end{array}$ & $\begin{array}{l}\text { Fragm. } \\
\text { G. mottes } \\
+ \text { cavités }\end{array}$ & $\begin{array}{l}\text { Massif } \\
\text { fissuré }\end{array}$ & $\begin{array}{c}\text { Massif } \\
\Gamma\end{array}$ & $\begin{array}{c}\text { Massif } \\
\Delta\end{array}$ & \\
\hline \multicolumn{7}{|c|}{ Traitement $\mathbf{O}$} \\
\hline G82 & 83 & 95 & $*$ & 66 & $*$ & 89 \\
\hline M82 & 79 & 74 & $*$ & 38 & $*$ & 80 \\
\hline G83 & 71 & 72 & $*$ & 48 & $*$ & 93 \\
\hline G85 & 83 & 74 & $*$ & $*$ & $*$ & 93 \\
\hline \multicolumn{7}{|c|}{ Traitement $\mathbf{B}$} \\
\hline G82 & $*$ & 86 & 50 & * & 5 & 63 \\
\hline M82 & $*$ & 75 & 60 & $*$ & 6 & 60 \\
\hline G83 & * & 81 & 66 & * & 7 & 30 \\
\hline \multicolumn{7}{|c|}{ Traitement C } \\
\hline G82 & $*$ & $*$ & 80 & 40 & $*$ & 79 \\
\hline M82 & $*$ & $*$ & 71 & $*$ & 7 & 20 \\
\hline G83 & $*$ & $*$ & 65 & $*$ & 8 & 29 \\
\hline G85 & $*$ & $*$ & 71 & $*$ & 3 & 12 \\
\hline
\end{tabular}

importante des racines n'est pas en contact avec la terre, et située dans des cavités structurales.

\section{b) Couches non travaillées}

Nous avons montré (TARDIEU \& MANICHON, 1986 $b$ ) sur les cartes racinaires horizontales effectuées à G83 que les obstacles de dimensions décimétriques situés dans la couche labourée créent un "effet d'ombre" dans les zones du sous-sol situées à la verticale, y causant ainsi des réductions localisées de la densité racinaire. Cet effet a également été observé à M82 (fig. 5) et à G85. Dans les couches non travaillées, la disposition des racines apparaît donc également plus groupée en $\mathrm{C}$ qu'en $\mathrm{O}, \mathrm{B}$ occupant une position proche de $\mathrm{O}$.

\section{Caractérisation synthétique des enracinements en tant que capteurs d'eau}

$\mathrm{Au}$ début du cycle G83, les valeurs de l'indice synthétique apparaissent significativement inférieures en $\mathrm{B}$ et $\mathrm{C}$ par rapport à $\mathrm{O}$ (fig. 6). Ceci est dû, dans le traitement $\mathrm{C}$, au faible développement vertical du système racinaire, et en $B$, au mauvais contact terreracine. A partir du stade $8 \mathrm{f}$, on constate une forte augmentation des valeurs de l'indice dans les traitements $\mathrm{O}$ et $\mathrm{B}$, liée à l'approfondissement du front racinaire, alors que les valeurs correspondant au traitement $\mathrm{C}$ augmentent plus lentement. Le classement $\mathrm{O}>\mathrm{B}>\mathrm{C}$ persiste jusqu'à la floraison, en liaison, dans ce dernier traitement, avec la disposition forte- 


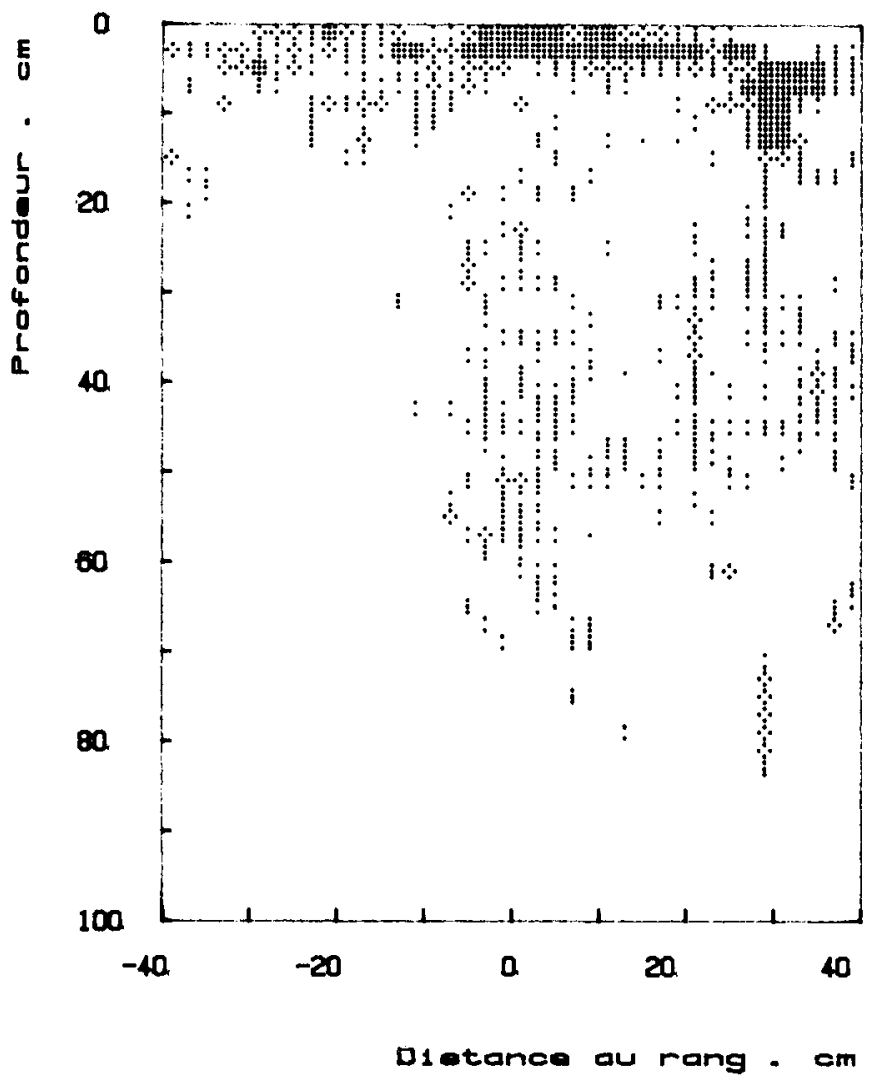

Figure 5

Cartographie verticale d'une placette du traitement $C$ (Montmirail 1982), présentant un exemple de "zone d'ombre " causée par un obstacle structural situé dans la couche labourée. (La note de densité racinaire correspondant à chaque case de la grille est Jigurée par un symbole d'autant plus foncé que la note est élevée.)

Vertical mapping, treatment $C$ (Montmirail 1982), showing an example of a « shadow zone 》 caused by a structural obstacle within the ploughed layer (root density index corresponding to each square of the grid figured progressively by darker symbols). ment groupée des racines. Le classement entre traitements est le même à G82, M82 et G85 (tabl. 5), la discrimination entre traitements étant plus nette pour cette variable que pour les variables Masse ou Profondeur d'enracinement.

Dans les traitements $\mathrm{O}$ et $\mathrm{C}$, où des cartographies racinaires ont été faites à la floraison de G83 sur 5 plans horizontaux, ces résultats sont confirmés par l'étude de la fonction de répartition des distances entre chaque point de ces plans et l'impact de racine le plus proche (fig. 7). Alors que dans le traitement $O$, la totalité des points se trouve à une distance inférieure à

\section{TABLEAU 5}

Valeurs à la floraison de l'indice synthétique de caractérisation de l'enracinement en tant que capteur d'eau.

Values at silking of the synthetic index for characterization of root system as a water sink.

\begin{tabular}{lccc}
\hline & \multicolumn{3}{c}{ Traitement } \\
\cline { 2 - 4 } & 0 & $\mathrm{~B}$ & $\mathrm{C}$ \\
\hline $\mathrm{G} 82$ & & & \\
$\mathrm{x}$ & $1356 \mathrm{a}$ & $1135 \mathrm{~b}$ & $1060 \mathrm{c}$ \\
$\mathrm{CV}$ & 7 & 8 & 9 \\
\hline $\mathrm{M} 82$ & & & \\
$\mathrm{x}$ & $1419 \mathrm{a}$ & $1233 \mathrm{a}$ & $1013 \mathrm{~b}$ \\
$\mathrm{CV}$ & 4 & 6 & 17 \\
\hline $\mathrm{G} 83$ & \multicolumn{4}{c}{} \\
$\mathrm{x}$ & $1569 \mathrm{a}$ & $1179 \mathrm{~b}$ & $731 \mathrm{c}$ \\
$\mathrm{CV}$ & 4 & 9 & 32 \\
\hline $\mathrm{G} 85$ & \multicolumn{4}{c}{} \\
$\mathrm{x}$ & 1614 & & 803 \\
$\mathrm{CV}$ & 6 & & - \\
\hline \hline
\end{tabular}

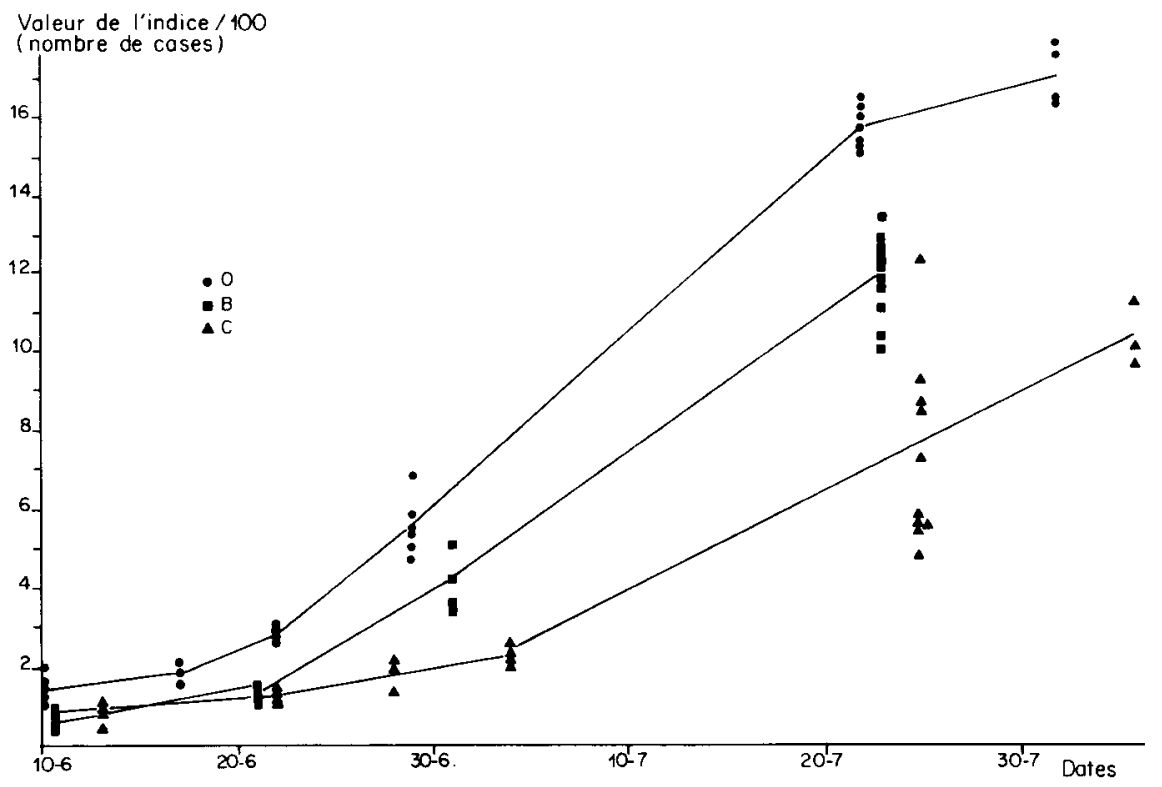

Figure 6

Evolution dans le temps de l'indice synthétique de caractérisation de l'enracinement en tant que capteur d'eau. Grignon 1983.
Change with time in the synthetic index of characterization of the root system as a water sink. Grignon 1983. 


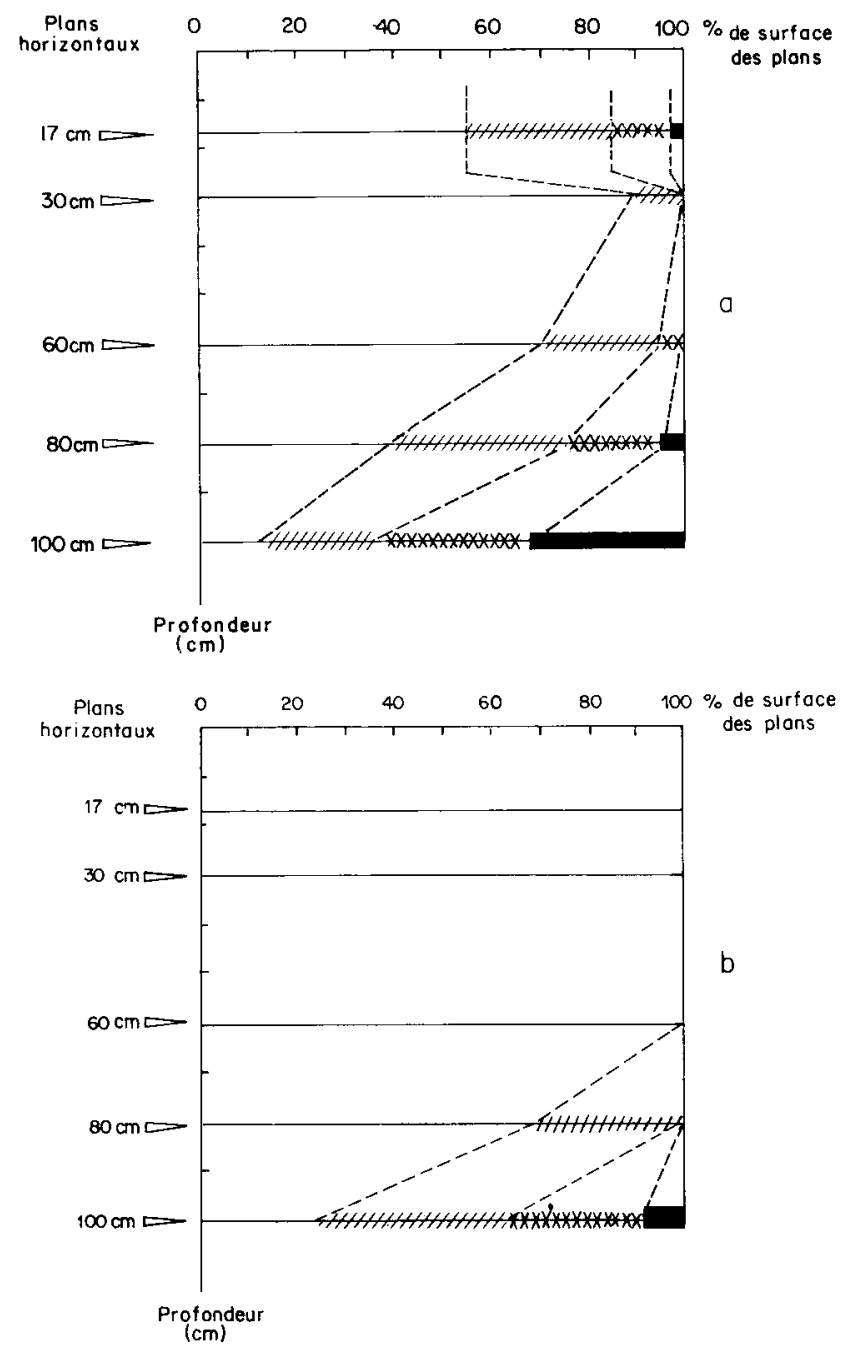

Figure 7

Fonction de répartition des distances entre chaque point du sol et la racine la plus proche, pour les 5 plans étudiés. Floraison, Grignon 1983.

$a:$ Traitement $C$

$b:$ Traitement $O$

(Le plan $17 \mathrm{~cm}$ est supposé représentatif de l'ensemble de la couche labourée.)

Cumulative frequency distribution of distances between each point of the soil and the nearest root, for the 5 horizontal planes. Silking, Grignon 1983.

$a:$ treatment $C$

$b:$ treatment $O$

(The $17-\mathrm{cm}$ deep plane is considered as representative of the whole ploughed layer.)

Points situés à moins de $4 \mathrm{~cm}$ d'un impact de racines.

Points situés entre 4 et $7 \mathrm{~cm}$ d'un impact.

$\times x \times x \times x$ Points situés entre 7 et $12 \mathrm{~cm}$ d'un impact.

Points situés à plus de $12 \mathrm{~cm}$ d'un impact.

$4 \mathrm{~cm}$ d'un impact jusqu'à $60 \mathrm{~cm}$ de profondeur, en $\mathrm{C}$ une proportion appréciable de points de cette même couche se trouve entre 4 et $12 \mathrm{~cm}$ de l'impact le plus proche.

\section{DISCUSSION}

\section{A. Colonisation de la couche labourée}

Les différences entre traitements de la masse racinaire ne deviennent significatives, à stade phénologique égal, qu'à partir de la montaison ; c'est aussi le cas pour la masse aérienne (TARDIEU, 1984). On peut rap- procher cela du fait que le premier milieu d'accueil des racines, l'horizon $\mathrm{H} 1$, est favorable à l'enracinement dans tous les traitements. La disposition spatiale des racines $\mathbf{a}$, au contraire, été affectée dès le début du cycle : l'approfondissement du front racinaire a été bloqué en C par l'obstacle continu que constitue l'horizon H5, jusqu'à la fin juin. Sa vitesse est ensuite liée à la dynamique de fissuration de cet horizon à G83, ce qui est également vraisemblable dans les autres situations expérimentales (où ces dynamiques n'ont pas été suivies), puisque seules les fentes de retrait ont été colonisées.

- Dans les traitements B et $\mathrm{C}$, les zones $\mathrm{M} \Delta$ ont été très peu pénétrées par les racines, bien que leur teneur en eau soit restée proche de la "capacité au champ " pendant une grande partie de la période de croissance des racines, et qu'elle soit plus élevée que dans les zones fragmentaires voisines (TARDIEU \& MANICHON, 1987). Ceci est vraisemblablement lié à la porosité réduite de ces mottes. Les mécanismes entrant en jeu sont la résistance mécanique à la pénétration (TAYLOR \& GARDNER, 1963 ; MAERTENS, $1964 b$; SCHUURMAN, 1965), et aussi vraisemblablement la faible circulation de l'oxygène (BARLEY, 1963 ; EAVIS, 1972). La porosité intramottière a, d'autre part, joué un rôle important sur la pénétration des racines : les zones massives $\Gamma$ de G82 ont été considérablement plus colonisées que les zones massives $\Delta$ dans la même situation.

\section{B. Colonisation des couches non travaillées}

D'autres mécanismes que la résistance mécanique à la pénétration doivent être invoqués pour expliquer les écarts entre traitements de colonisation de l'horizon $\mathrm{H} 1$ et des couches non travaillées. En effet, l'état structural et la teneur en eau du sol (en début d'essai) y étaient invariants entre traitements (TARDIEU \& MANICHON, 1987) ; cette dernière est devenue par la suite supérieure en $\mathrm{C}$ par rapport à $\mathrm{O}$ et $\mathrm{B}$.

1) Les études sur plans horizontaux (TARDIEU \& MANICHON, 1986 $b$ ) et l'observation des cartes racinaires verticales montrent l'existence d'un "effet d'ombre " des obstacles à la pénétration des racines situés dans la couche labourée. Ce phénomène est vraisemblablement lié aux modalités d'implantation du système racinaire. Les racines primaires qui colonisent les couches non travaillées ont pris des trajets principalement verticaux, d'une part à cause du géotropisme, d'autre part du fait qu'elles ont emprunté des galeries de vers de terre et des fissures verticales. Des obstacles situés dans la couche labourée, qui empêchent la progression verticale des racines, provoquent ainsi leur absence complète dans les zones situées sous l'obstacle (effet d'" ombre "). Des "zones d'ombre " ont été constatées dans le traitement $C$ de M82, G83 et 85 , où des zones massives $\Delta$ de dimensions décimétriques existent dans l'horizon $\mathrm{H} 5$, causant un groupement très marqué des racines. Dans les situations où le groupement est moins marqué dans cet horizon, ce qui est le cas en B (obstacles centimétriques) et en C de G82 (état interne $\Gamma$ ), cet effet n'a pas été observé. Pour s'extérioriser, il nécessite donc probablement la présence d'obstacles décimétriques à la pénétration des racines dans la couche labourée. 
2) Cet «effet d'ombre » ne peut donc pas rendre compte des réductions de densité racinaire dans les couches non travaillées en C de G82 et en B de toutes les situations, par rapport à O. D'autre part, s'il modifie la disposition spatiale des racines, cet effet ne permet pas d'expliquer simplement les différences de colonisation de ces couches prises dans leur totalité. L'explication la plus plausible nous parait être la différence entre traitements de vitesse de croissance, qui s'est manifestée à partir de la montaison tant pour le système racinaire (fig. 1) que pour les parties aériennes (TARDIEU, 1984). A la floraison, des relations linéaires étroites existent entre la masse aérienne, la masse racinaire totale et la densité racinaire dans les couches non travaillées. Les différences de croissance entre traitements se sont donc répercutées sur les parties aériennes, mais aussi sur les parties souterraines, y compris celles situées dans les couches non travaillées.

Parmi les hypothèses qui peuvent être avancées pour expliquer les différences de vitesse de croissance entre traitements, nous pouvons mentionner celle qui fait appel à la capacité du système racinaire à capter l'eau du sol. Si le corps d'hypothèses exposées dans un article précédent (TARDIEU \& MANICHON, 1986a) est juste, les systèmes racinaires des traitements $B$ et $C$ ont eu à chaque instant une moindre capacité à capter l'eau par rapport à $\mathrm{O}$, en raison de leur disposition spatiale et du contact sol-racine (fig. 6 et 7). Cette différence de « qualité » de capteur, existant dès les premières phases du cycle, s'est répercutée sur l'alimentation hydrique et la vitesse de croissance des peuplements végétaux.

Cette hypothèse sera testée dans le prochain article de cette série, où les classements entre traitements établis d'après l'étude de l'enracinement seront confrontés à des mesures hydriques.concernant les mêmes peuplements. Elle n'est pas exclusive : tout facteur provoquant des écarts de vitesse de croissance entre traitements (absorption d'azote ou vitesse de réchauffement du sol, par exemple) pourrait contribuer à expliquer les différences de colonisation racinaire entre les traitements expérimentaux.

\section{Méthodes de caractérisation de l'enracinement uti- lisées sur ce dispositif (cartographie verticale, cartographies horizontales, endoscopie)}

Ces résultats soulignent l'intérêt de l'étude de la dynamique d'approfondissement du front racinaire et de celle de la disposition spatiale des racines, alors que ces 2 caractéristiques sont difficilement prises en compte par les méthodes classiques d'étude du système racinaire (TARDIEU \& MANICHON, 1986a). Trois observations supplémentaires peuvent être faites ici :

1) Les coefficients de variation obtenus pour les variables étudiées sont relativement bas, s'agissant de variables caractérisant l'enracinement. Cela est probablement dû au fait que les hétérogénéités centimétriques à décimétriques de la densité racinaire ne sont ici pas prises en compte dans ces $C V$, à cause du mode de traitement des cartes racinaires (TARDIEU \& MANICHON, 1986b). Elles le sont, au contraire, dans les autres méthodes de caractérisation où les échantillons ont généralement un volume de l'ordre du $\mathrm{dm}^{3}$. La fai- blesse des $\mathrm{CV}$ tient aussi aux précautions prises afin de limiter la variabilité spatiale de l'état structural de la couche labourée à l'intérieur de chaque traitement (TARDIEU \& MANICHON, 1987).

2) L'indice synthétique établi à partir des cartes verticales fournit des résultats semblables au calcul sur 5 plans horizontaux de la fonction de répartition des distances entre chaque point du sol et la racine la plus proche. Son obtention nécessite un temps d'observation sur le terrain environ 3 fois plus court. Bien qu'il ne fournisse pas d'estimation précise des distances, puisqu'il est établi à partir de cartes verticales (TARDIEU \& MANICHON, 1986a), il pourrait être, au moins dans certains types de sols, un outil utile de comparaison entre systèmes racinaires.

3) Avec le mode d'implantation que nous avons adopté pour les tubes d'accès de l'endoscope, la perturbation liée à l'interface sol-tube rend délicate l'interprétation des observations endoscopiques. Cette perturbation a, en particulier, modifié localement la dynamique d'approfondissement $\mathrm{du}$ front racinaire, notamment dans le traitement $C$. D'autre part, dans tous les traitements, les densités surfaciques de racines observées à travers les tubes étaient considérablement plus élevées que celles observées sur les plans horizontaux (colonisation préférentielle de l'interface sol-tube). Une amélioration du mode d'implantation des tubes est donc une condition nécessaire au développement des techniques endoscopiques.

\section{CONCLUSION}

Les types d'états structuraux que nous avons définis dans le premier article de cette série ont entraîné une croissance et une disposition spatiale très différenciées du système racinaire. Les réactions observées ont été relativement stables dans les 4 situations expérimentales étudiées. Une analyse rapide aurait pu conduire à interpréter les différences entre traitements comme le seul effet de la résistance mécanique du sol à la pénétration ; nos résultats seraient alors une généralisation au champ de ceux obtenus au laboratoire par de nombreux auteurs. Nous avons, au contraire, montré que, pour étudier les relations entre l'état structural de la couche labourée et l'enracinement, il est nécessaire de prendre en compte plusieurs types de mécanismes :

- Les relations entre l'état physique du sol et la pénétration des racines expliquent les différences, entre traitements, de disposition spatiale des racines dans la couche labourée. Cette première relation est une application directe des modèles cités en introduction.

- Des relations mécaniques plus complexes, mettant en cause la direction privilégiée des pores dans les couches non travaillées, permettant d'interpréter l' « effet d'ombre " des obstacles à l'enracinement situés dans la couche labourée.

- Enfin, les écarts de vitesse de croissance du système racinaire, y compris dans les couches non travaillées, sont vraisemblablement dues à des différences de fonctionnement des peuplements végétaux. Celles-ci sont liées, notamment, à la capacité du système racinaire à extraire à chaque instant l'eau et les éléments minéraux du sol. 
Seules les 2 dernières relations évoquées permettent de comprendre les écarts de colonisation racinaire dans les couches non affectées par les tassements. Nous pensons que leur prise en compte est nécessaire, d'une part pour l'extrapolation à des milieux différents de ceux que nous avons étudiés, d'autre part pour entreprendre la modélisation de la croissance et de la disposition spatiale des racines.

\section{REMERCIEMENTS}

Les auteurs remercient J. CANEill pour l'aide apportée lors du traitement des données, ainsi que la société LiMAGRAIN qui a financé une partie de ce travail.

Reç le 25 novembre 1985. Accepté le 28 novembre 1986.

\section{RÉFÉRENCES BIBLIOGRAPHIQUES}

\begin{abstract}
Allmaras R. R., Nelson W. W., Voorhees W. B., 1975. Soybean and corn rooting in southwestern Minnesota. II. - Root distributions and related water inflow. Soil Sci. Soc. Am. Proc., 39, 771-777.
\end{abstract}

Aubertin G. M., Kardos L. T., 1965. Root growth through porous media under controlled conditions. I. - Effect of pore size and rigidity. Soil Sci. Soc, Am. Proc., 29, 290-293.

Barber S. A., 1971. Effect of the tillage practice on corn root distribution and morphology. Agron. J., 63, 724-726.

Barley K. P., 1963. Influence of soil strength on growth of roots. Soil Sci., 96, 175-180.

Brouwer R., 1966. Root growth of grasses and cereals. In : "The growth of cereals and grasses " (Eds. F. L. Milthorpe and J. D. Ivins), 153-156, Butterworth, London, 358 p.

Dexter A. R., Hewitt J. S., 1978. The deflection of plant roots. $J$. agric. Eng. Res., 23, 17-22.

Eavis B. W., 1972. Soil physical conditions affecting seedling root growth. I. - Mechanical impedance, aeration and moisture availability as influenced by bulk density and moisture levels in a sandy loam soil. Plant Soil, 36, 613-622.

Foth H. D., 1962. Root and top growth of corn. Agron. J., 54, 4952.

Hewitt J. S., Dexter A. R., 1984. The behaviour of roots encountering cracks in soil. II. - Development of a predictive model. Plant Soil, 79, 11-28.

Jordan M. O., 1986. Mise en place du système racinaire du maïs. Définition et caractérisation au champ et en conditions contrôlées des paramètres descriptifs de la structure racinaire. Thèse Strasbourg I, $177 \mathrm{p}$.

Lubet E., Juste C., 1985. Cinétique de la production de matière sèche et de prélèvement d'éléments nutritifs par une culture irriguée de maïs à haute potentialité de rendement. Agronomie, 5, 329-350.

Maertens C., 1964a. Influence des propriétés physiques des sols sur le développement radiculaire et conséquences sur l'alimentation hydrique et azotée des cultures. Sci. Sol, 2, 31-41.

Maertens C., 1964b. La résistance mécanique des sols à la pénétration : ses facteurs et son influence sur l'enracinement, Ann. agron., 15, 539-554.

Maertens C., Clauzel Y., 1982. Premières observations sur l'utilisation de l'endoscopie dans l'étude de l'enracinement in situ de plantes cultivées. Agronomie, 2, 677-680.

Mengel D. B., Barber S. A., 1974. Development and distribution of the corn root system under field conditions. Agron. J., 66, 341-344.
Picard D., Jordan M. O., Trandel R., 1985. Rythme d'apparition des racines primaires du maïs (Zea mays L.). I. - Etude détaillée pour une variété en un lieu donné. Agronomie, 5 (8), 667-676.

Raghavan G. S. V., Mc Kyes E., Baxter R., Gendron G., 1979. Trafic-soil-plant (maize) relations. J. Terramechanics, 16, 181-189.

Schuurman J. J., 1965. Influence of soil density on root development and growth of oats. Plant Soil, 22, 352-374.

Scott-Russel R., Goss M. J., 1974. Physical aspects of soil fertility. The response of roots to mechanical impedance. Neth. J. agric. Sci., 22, 305-318.

Sébillotte M., 1963. Action de la structure du sol sur une culture de féverole. C. R. Acad. Agric. Fr., 305-318.

Tardieu F., 1984. Etude au champ de l'enracinement du mais. Influence de l'état structural sur la répartition des racines. Conséquences pour l'alimentation hydrique. Thèse Doct. Ing. INA-PG, Paris, $232 \mathrm{p}$

Tardieu F., Manichon H., 1986a. Caractérisation en tant que capteur d'eau de l'enracinement du maîs en parcelle cultivée. I. - Discussion des critères d'étude. Agronomie, 6 (4), 345-354.

Tardieu F., Manichon H., 1986b. Caractérisation en tant que capteur d'eau de l'enracinement du maïs en parcelle cultivée. II. - Une méthode d'étude de la répartition verticale et horizontale des racines. Agronomie, 6 (5), 415-425.

Tardieu F., Manichon H., 1987. Etat structural, enracinement et alimentation hydrique du maiis. I. - Modélisation d'états structuraux types de la couche labourée. Agronomie, 7 (2), 123-131.

Tardieu F. Etat structural, enracinement et alimentation hydrique du maïs. III. - Disponibilité des réserves en eau du sol. Agronomie, 7 (4).

Taylor H. M., Gardner H. R., 1963. Penetration of cotton seedling taproots as influenced by bulk density, moisture content and strength of soil. Soil Sci., 96, 153-156.

Varlet-Grancher C., 1982. Analyse du rendement de la conversion de l'énergie solaire pour un couvert végétal. Thèse d'état, Université Paris-Sud, $\mathrm{n}^{\circ} 2593,144 \mathrm{p}$.

Welbank P. J., Gibb M. J., Taylor P. J., Williams E. D., 1974. Root growth of cereal crops. Rep. Rothamsted Exp. St. for 1973, 2 , 22-26.

Wiersum L. K., 1957. The relationship of the size and structural rigidity of pores to their penetration by roots. Plant Soil., 9, 75-85. 